ARTICLE

https://doi.org/10.1038/s41467-019-10519-z

\title{
Impact of polymorphism on the optoelectronic properties of a low-bandgap semiconducting polymer
}

Mengmeng Li (10 1,2, Ahmed Hesham Balawi ${ }^{3}$, Pieter J. Leenaers' ${ }^{1}$, Lu Ning ${ }^{4}$, Gaël H.L. Heintges ${ }^{1,5}$, Tomasz Marszalek 6,7, Wojciech Pisula6,7, Martijn M. Wienk1, Stefan C.J. Meskers (D) ${ }^{1}$, Yuanping Yi(D) ${ }^{4}$, Frédéric Laquai id ${ }^{3}$ \& René A.J. Janssen (1) ${ }^{1,2}$

Polymorphism of organic semiconducting materials exerts critical effects on their physical properties such as optical absorption, emission and electrical conductivity, and provides an excellent platform for investigating structure-property relations. It is, however, challenging to efficiently tune the polymorphism of conjugated polymers in aggregated, semi-crystalline phases due to their conformational freedom and anisotropic nature. Here, two distinctly different semi-crystalline polymorphs $\left(\beta_{1}\right.$ and $\beta_{2}$ ) of a low-bandgap diketopyrrolopyrrole polymer are formed through controlling the solvent quality, as evidenced by spectroscopic, structural, thermal and charge transport studies. Compared to $\beta_{1}$, the $\beta_{2}$ polymorph exhibits a lower optical band gap, an enhanced photoluminescence, a reduced $\pi$-stacking distance, a higher hole mobility in field-effect transistors and improved photocurrent generation in polymer solar cells. The $\beta_{1}$ and $\beta_{2}$ polymorphs provide insights into the control of polymer self-organization for plastic electronics and hold potential for developing programmable ink formulations for next-generation electronic devices.

\footnotetext{
${ }^{1}$ Molecular Materials and Nanosystems, Institute for Complex Molecular Systems, Eindhoven University of Technology, P.O. Box 513, 5600 MB Eindhoven, The Netherlands. ${ }^{2}$ Dutch Institute for Fundamental Energy Research, De Zaale 20, 5612 AJ Eindhoven, The Netherlands. ${ }^{3}$ KAUST Solar Center (KSC), Physical Sciences and Engineering Division (PSE, Material Science and Engineering Program (MSE)), King Abdullah University of Science and Technology (KAUST), Thuwal 23955-6900, Saudi Arabia. ${ }^{4}$ Beijing National Laboratory for Molecular Science, Key Laboratory of Organic Solids, Institute of Chemistry, Chinese Academy of Sciences, 100190 Beijing, People's Republic of China. ${ }^{5}$ Institute for Materials Research (IMO-IMOMEC), Design and Synthesis of Organic Semiconductors (DSOS), Hasselt University, Agoralaan, 3590 Diepenbeek, Belgium. ${ }^{6}$ Max Planck Institute for Polymer Research, Ackermannweg 10, 55128 Mainz, Germany. 7 Faculty of Chemistry, Department of Molecular Physics, Lodz University of Technology, Zeromskiego, 116, 90-924 Lodz, Poland. Correspondence and requests for materials should be addressed to R.A.J.J. (email: r.a.j.janssen@tue.nl)
} 
olymorphism is the ability of a compound to adopt multiple packing motifs in the solid or aggregated state and has important consequences in chemistry, physics, material science, and biology ${ }^{1}$. Among organic semiconducting materials, considerable progress in understanding polymorphism has been made using single crystals of conjugated small molecules ${ }^{2-6}$. It was found that a slight change in crystal packing critically affects the charge transport in electronic devices ${ }^{6}$. Traditionally the structure-property relations of organic semiconductors are mainly studied via chemical modifications such as regioregularity control and side chain engineering ${ }^{7,8}$. In comparison, polymorphs provide an ideal platform for such investigations by excluding the influence of chemical modification. Polymorphism of conjugated semiconducting polymers has been reported for few polymers $^{9-17}$.

In most conjugated polymers, an amorphous "spaghetti-like" phase $(\alpha)$ typically coexists with an aggregated phase $(\beta)$ composed of relatively ordered crystalline regions ${ }^{18}$. The $\beta$ phase originates from strong intermolecular interactions between functional units or aromatic rings in the polymer backbone ${ }^{19}$. The transition between the amorphous $\alpha$ and semi-crystalline $\beta$ phases of conjugated polymers, such as in polyfluorenes, can be realized via thermal treatment ${ }^{20}$ or by using mixtures of good/ poor solvents ${ }^{21-23}$. A comprehensive investigation of polymorphism of the semi-crystalline $\beta$ phases of conjugated polymers has not yet been achieved. One prominent example that grants access to two different semi-crystalline polymorphs is formed by poly(3-alkylthiophene)s, whose solid state structure can be controlled by the evaporation rate of solvent ${ }^{24}$, exposure to solvent vapors ${ }^{25}$, molecular weight ${ }^{26}$, and thermal annealing ${ }^{27}$. The first polymorph found in most occasions exhibits an end-toend packing of the lamellae, while the second one has interdigitated side chains with a reduced lamellar spacing, as confirmed using X-ray measurements ${ }^{28}$. From the aspect of spectroscopy, two H-type aggregates in solution were identified at extremely low temperature for poly(3-hexylthiophene) by Franck-Condon analyses of emission spectra ${ }^{29}$. Another example of polymorphism has been found for the naphthalene diimide based copolymer (P(NDI2OD-T2)) that shows an extra absorption peak at longer wavelengths when dissolved in a poor solvent, indicating the formation of a secondary aggregated phase ${ }^{19}$. Other signs of polymorphism were found for conjugated polymers, blended with polyethylene oxide (PEO) which afforded differences in the optical absorption before and after treatment with water. ${ }^{30}$ Generally, the magnitude of the changes in optoelectronic or thermal properties depends on the structural differences between the polymorphs. Polymorphism in aggregated phases can also be obtained in solid films, but post-treatment or particular growth methods are prerequisites 9,19 . However, these reports lack detailed spectroscopic and structural characterizations, which could reveal the role of polymorphism in the structure-property relations and, more importantly, in the optoelectronic property in electronic devices.

In this contribution, two aggregated, semi-crystalline phases $\left(\beta_{1}\right.$ and $\beta_{2}$ ) of a diketopyrrolopyrrole (DPP) based polymer ${ }^{31}$ are generated by precisely tuning the solvent quality. Both polymorphs can be formed in solution and in the solid state. The $\beta_{2}$ phase possess a lower optical band gap, demonstrating a more delocalized wave function. The steady-state and time-resolved photoluminescence $(\mathrm{PL})$ of polymer solutions reveal that the three phases, namely $\alpha, \beta_{1}$ and $\beta_{2}$, can coexist with excited state lifetimes of $29 \mathrm{ps}, 78 \mathrm{ps}$, and $129 \mathrm{ps}$, respectively. The extended chain conformation of the newly induced $\beta_{2}$ phase in solution is retained in thin films, as confirmed by both UV-vis-NIR absorption and PL measurements. Compared to $\beta_{1}$, the $\beta_{2}$ phase possesses a slightly smaller $\pi$-stacking distance but has identical lamellar spacing. The field-effect mobility of the $\beta_{2}$ phase is higher than that of $\beta_{1}$, likely due to the reduced $\pi-\pi$ stacking. In bulk heterojunction solar cells, a clear contribution of the $\beta_{2}$ polymorph to the external quantum efficiency (EQE) is observed via a long-wavelength peak. This study proposes a simple approach for tuning the polymorphism of conjugated polymers in both solution and solid state, and the characterization provides insights into the structure-property relation of nextgeneration devices for plastic electronics.

\section{Results}

Polymorphism in solution. DPP based polymers are a versatile class of organic semiconducting materials, whose optical properties can be manipulated from small $(1.13 \mathrm{eV})$ to wide band gaps $(1.77 \mathrm{eV})$ by modifying the aromatic substituents and $\pi-$ conjugated segments ${ }^{32-34}$. Typically, the UV-vis-NIR absorption spectra of DPP based polymers exhibit an intense first (0-0) vibronic absorption band and a less intense vibronic progression at shorter wavelengths (Supplementary Fig. 1), indicative of J-type aggregates $^{35}$. As shown in Fig. 1a, the absorption spectrum of DPDPP4T-HD shows $0-0$ and $0-1$ vibronic peaks in dilute chloroform (CF) solution at 770 and $700 \mathrm{~nm}$, respectively, together with a band at $424 \mathrm{~nm}$ due to a higher energy electronic state. Compared to the amorphous phase (denoted as $\alpha$ ) in which the polymer is molecularly dissolved, this aggregated phase is termed $\beta_{1}$. The intensity of the $0-0$ peak gradually decreases with the addition of 1,2,4-trichlorobenzene (TCB) to the CF solution, without affecting the $0-1$ peak. With increasing TCB content, the steady-state photoluminescence (PL) spectra reveal a reduced emission of the $\beta_{1}$ phase at $816 \mathrm{~nm}$, with a simultaneous increase of the emission of the $\alpha$ phase at $744 \mathrm{~nm}$. From the PL spectra we determine that in pure CF the $\alpha$ phase concentration is negligible because only $\beta_{1}$ emission is observed with excitation at $700 \mathrm{~nm}$, and emission of $\alpha$ phase at $740-750 \mathrm{~nm}$ is absent (black line in upper panel of Fig. 1b). These changes suggest a transition from the ordered $\beta_{1}$ phase in $\mathrm{CF}$ to the amorphous $\alpha$ phase upon adding TCB. When the CF:TCB ratio decreases to $1: 1$, the intensity of the $0-0$ absorption peak becomes identical to that of the $0-1$ peak. Surprisingly, when the CF:TCB ratio reaches 1:9, a completely new absorption peak emerges at $870 \mathrm{~nm}$, accompanied by an additional emission peak at $890 \mathrm{~nm}$ (Fig. 1a, b). Such change in both absorption and PL points to the formation of a second aggregated phase $\left(\beta_{2}\right)$. The red-shifted absorption and emission of the $\beta_{2}$ phase suggest that its wave functions are more delocalized, either by a decrease of intrachain disorder or by enhanced interchain interactions. Upon increasing the temperature of solutions containing mainly the $\beta_{1}$ phase or $\beta_{2}$ phase, their UV-vis-NIR absorption spectra exhibit an initial blue shift of the onset of absorption (Supplementary Fig. 2), followed by transformation into a featureless absorption band in a narrow temperature range, characteristic of the $\alpha$ phase in which intermolecular interactions are absent, followed at still higher temperature by a gradual spectral shift to lower wavelengths that can be interpreted as being the result of increased conformational freedom (interring rotation) of the polymer backbone. ${ }^{23,36,37}$

To quantify the phase evolution, the UV-vis-NIR absorption spectra recorded for D-PDPP4T-HD solutions as function of CF: TCB ratio are deconvoluted using multivariate curve resolution alternating least squares (MCR-ALS) analysis, as shown in Fig. 1c and Supplementary Fig. 3. By gradually adding TCB to CF from 0 vol.\% (1:0) to 66.7 vol.\% (1:2), the concentration of the $\beta_{1}$ phase continuously decreases, accompanied by a simultaneous increase of the $\alpha$ phase contribution, demonstrating the $\beta_{1} \rightarrow \alpha$ transformation (process I in Fig. 1e). The enhanced emission of $\alpha$ (Fig. 1b) confirms this $\beta_{1} \rightarrow \alpha$ transition. Eventually, more TCB 
a

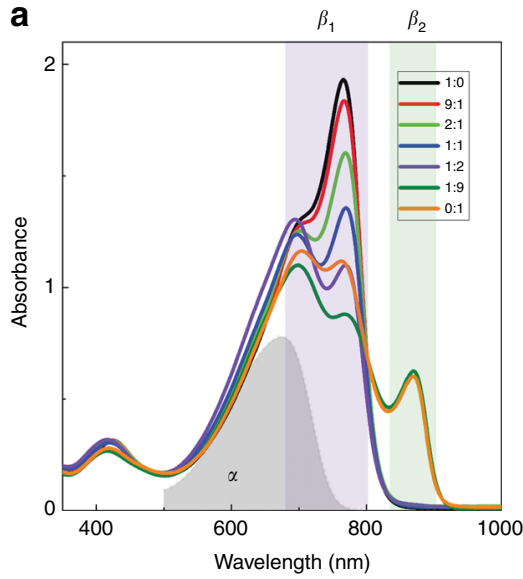

d

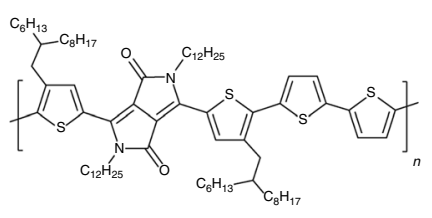

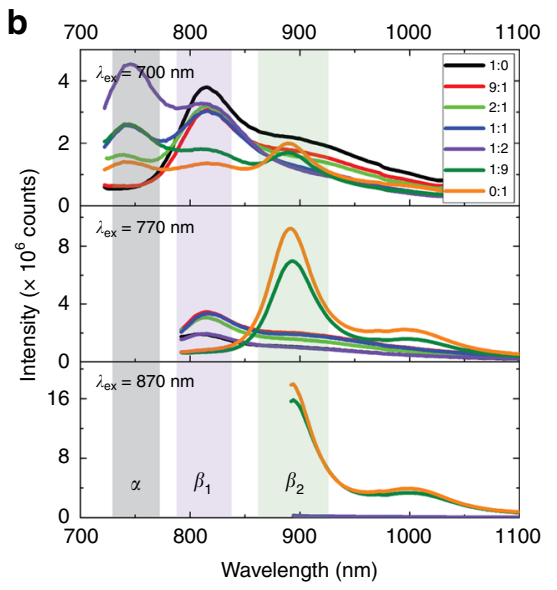

C

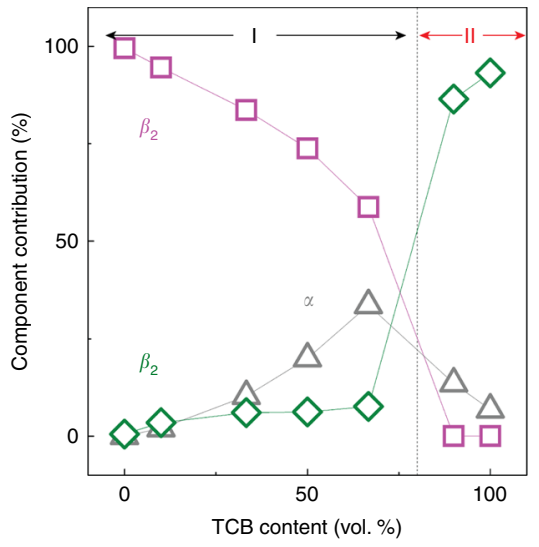

e
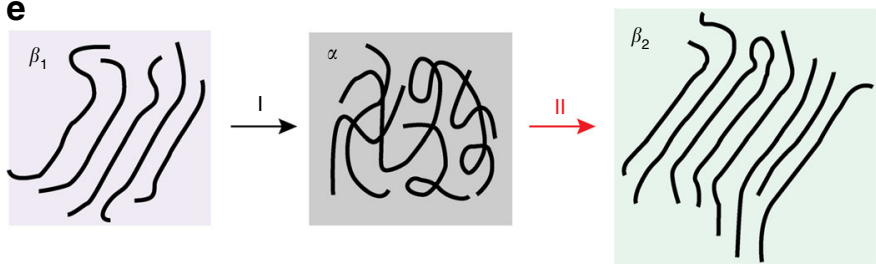

Fig. 1 Optical absorption and steady-state photoluminescence spectra in mixed solvents. $\mathbf{a}$, b UV-vis-NIR absorption and steady-state photoluminescence spectra of D-PDPP4T-HD dissolved in chloroform:1,2,4-trichlorobenzene (CF:TCB) mixtures. The volume ratio (v/v) of CF and TCB is 1:0, 9:1, 2:1, 1:1, 1:2, $1: 9$, and $0: 1$, respectively. The polymer concentration is $0.4 \mu \mathrm{M}$ in all cases. The gray area in a represents the absorption spectrum of the amorphous phase $(\alpha)$ in pure TCB at $100^{\circ} \mathrm{C}$ (Supplementary Fig. 2). The characteristic peaks of the first $\left(\beta_{1}\right)$ and the second $\left(\beta_{2}\right)$ aggregated phases are indicated by purple and green frames, respectively. c The component contribution of each phase as a function of the TCB content, as inferred from the multivariate curve resolution alternating least squares deconvolution of the UV-vis-NIR spectra. Process I in $\mathbf{c}$ indicates the $\beta_{1} \rightarrow \alpha$ transformation when TCB content

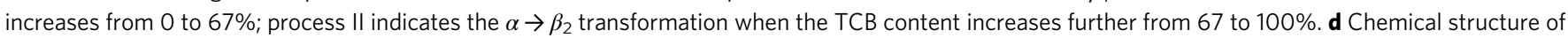
D-PDPP4T-HD. e Schematic illustration of polymorphism transitions of D-PDPP4T-HD

(1:9 and $0: 1)$ reduces the contents of the $\alpha$ and $\beta_{1}$ phases to the point that they almost cease to be present, while the new $\beta_{2}$ phase appears. At the highest TCB concentrations (1:9 and 0:1), the $\beta_{2}$ phase dominates and only emission of the $\beta_{2}$ phase is observed in the PL spectra (Fig. 1b).

To study the luminescence properties of the three polymorphs of D-PDPP4T-HD, we performed time-resolved photoluminescence (TRPL) measurements on dilute CF:TCB solutions. First, the two aggregated phases, $\beta_{1}$ and $\beta_{2}$, are investigated using excitation at $800 \mathrm{~nm}$, i.e., without exciting the $\alpha$ phase. Fig. $2 \mathrm{a}$ confirms that in the pure CF and TCB only one phase is present, i.e., only $\beta_{1}\left(\lambda_{\mathrm{em}}=825-840 \mathrm{~nm}\right)$ for $1: 0$ and only $\beta_{2}\left(\lambda_{\mathrm{em}}=\right.$ $870-890 \mathrm{~nm}$ ) for $0: 1$. The corresponding PL kinetics are fitted with mono-exponential decays, and fluorescence lifetimes of $78 \mathrm{ps}$ for $\beta_{1}$ and $129 \mathrm{ps}$ for $\beta_{2}$ (Fig. 2b) are determined. The PL lifetime of $\alpha$ phase $(29 \mathrm{ps})$ was determined in a TCB solution at $100^{\circ} \mathrm{C}$ (Supplementary Fig. 4).

The possibility of interaction between the two aggregated phases, $\beta_{1}$ and $\beta_{2}$, was investigated by time-resolved PL experiments at the onset of $\beta_{2}$ formation. At a ratio of 3:7 (70\% TCB) only a tiny amount of $\beta_{2}$ is seen in the UV-vis-NIR absorption, and $\beta_{1}$ is the dominant aggregated phase (Supplementary Fig. 5). At this critical ratio of 3:7, both emission peaks of $\beta_{1}$ and $\beta_{2}$ are clearly observed in the PL spectrum (Fig. 2a). In spite of the minor population of $\beta_{2}$ at the 3:7 ratio, the PL intensities of $\beta_{1}$ and $\beta_{2}$ are almost the same in the first picoseconds after excitation. This is a consequence of the fact that the PL quantum yield of $\beta_{2}$ is more than 30 -fold higher than that of $\beta_{1}$ (Supplementary Fig. 6 and Supplementary Note 1). At the 3:7 ratio, average PL lifetimes of $68 \mathrm{ps}\left(\lambda_{\mathrm{em}}=835 \mathrm{~nm}\right)$ for $\beta_{1}$ and $125 \mathrm{ps}\left(\lambda_{\mathrm{em}}=880 \mathrm{~nm}\right)$ for $\beta_{2}$ are found using bi-exponential fits (Supplementary Fig. 7 and Supplementary Table 1). These values are almost identical to the PL lifetimes of the pure aggregated phases, indicating that there is no, or very little, excited state energy transfer from $\beta_{1}$ to $\beta_{2}$. This indicates that the transformation of $\beta_{1}$ into $\beta_{2}$ does not occur within the aggregated phase (i.e., $\beta_{1} * \beta_{2}$ ), because in such case the close contact between the two phases should result in excited state energy transfer. Instead, the $\beta_{2}$ phase is formed via the molecularly dissolved amorphous $\alpha$ phase (i.e., $\beta_{1} \rightarrow \alpha \rightarrow \beta_{2}$ ). This is also supported by the temperature dependent UV-vis-NIR absorption spectra that show $\beta_{1} \rightarrow \alpha$ and $\beta_{2} \rightarrow \alpha$ transitions, but no indication of a $\beta_{2} \rightarrow \beta_{1}$ transformation (Supplementary Fig. 2). Further experiments for different solvent ratios (Supplementary Figs. 7, 8, and 9 and Supplementary Tables 2 and 3) also did not reveal any sign of energy transfer among $\alpha, \beta_{1}$ and $\beta_{2}$. This indicates that in solution the phases are beyond the Förster radius (on the order of $5 \mathrm{~nm}$ ) of each other.

Having established the existence of $\beta_{1}$ and $\beta_{2}$, we sought to reveal the origin of the two aggregated phases. To this aim we employ molecular dynamics simulations for a D-PDPP4T-HD nonamer in $\mathrm{CF}$ and TCB (Fig. 3a), following the protocol described in ref. ${ }^{38}$. The simulation window between 20 and 30 ns is selected for data sampling, because at $20 \mathrm{~ns}$ thermal equilibrium has been achieved (Supplementary Fig. 10). In particular, we analyze the conformation of the conjugated chain (main chain), the branched side chain on the thiophene unit (side chain I) and the linear side chain on the DPP unit (side chain II) (Fig. 3b, c). The size of the nonamer is quantitatively evaluated by its gyration radius $\left(R_{\mathrm{g}}\right)$ and by the distances between the heads and the tails $\left(d_{\mathrm{t}}\right)$ of the main and side chains, as shown in Fig. 3d-o. Compared to $\mathrm{CF}$, the average $R_{\mathrm{g}}$ of the main chain in TCB is noticeably 
a
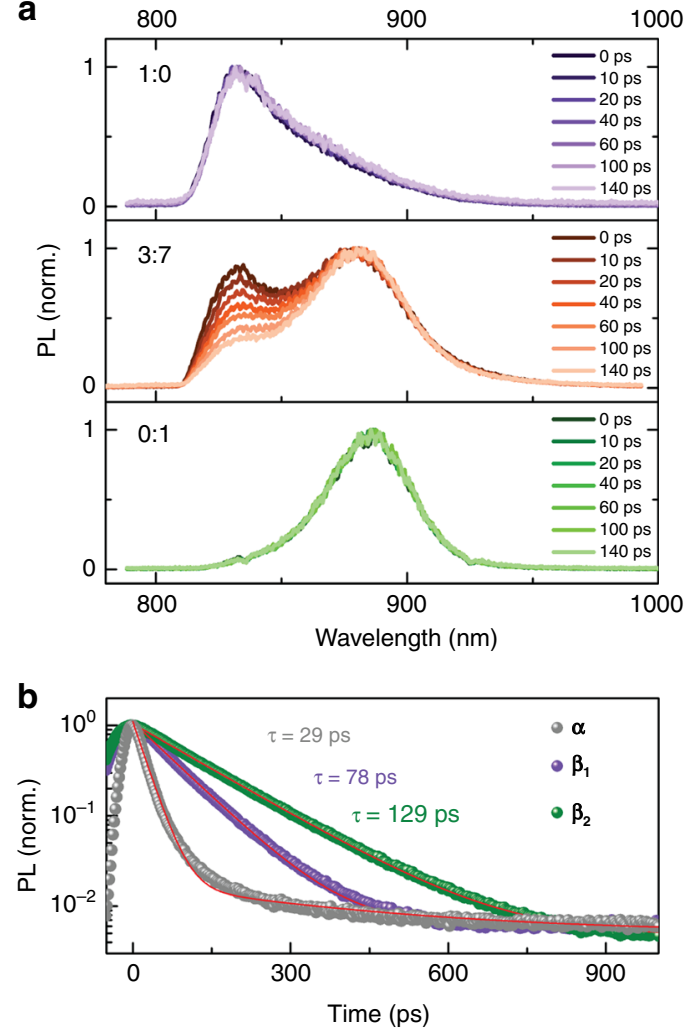

Fig. 2 Time-resolved photoluminescence in mixed solvents. a Normalized time-resolved photoluminescence (TRPL) spectra recorded at various time delays for a solution of D-PDPP4T-HD in 1:0, 3:7 and 0:1 ( $\mathrm{v} / \mathrm{v})$ of chloroform:1,2,4-trichlorobenzene (CF:TCB). The polymer concentration is $0.4 \mu \mathrm{M}$. Excitation was performed at $800 \mathrm{~nm}$ with $80 \mathrm{MHz}$ laser pulse repetition rate. A $808 \mathrm{~nm}$ long-pass filter was used to suppress the scattered laser pulse. $\mathbf{b}$ Emission dynamics of $\alpha$ phase from 0:1 solution at $100^{\circ} \mathrm{C}$ with $\lambda_{\mathrm{ex}}=400 \mathrm{~nm}$ and $\lambda_{\mathrm{em}}=815-865 \mathrm{~nm}, \beta_{1}$ phase from 1:0 solution with $\lambda_{\mathrm{ex}}=800 \mathrm{~nm}$ and $\lambda_{\mathrm{em}}=825-840 \mathrm{~nm}$, and $\beta_{2}$ phase from 0:1 solution with $\lambda_{\mathrm{ex}}=800 \mathrm{~nm}$ and $\lambda_{\mathrm{em}}=870-890 \mathrm{~nm}$ enhanced from $4.20 \mathrm{~nm}$ to $4.58 \mathrm{~nm}$. Moreover, the average value of the main chain $d_{\mathrm{t}}$ is also increased from $13.11 \mathrm{~nm}$ in CF to $15.36 \mathrm{~nm}$ in TCB. The increase in both $R_{\mathrm{g}}$ and $d_{\mathrm{t}}$ indicates a more extended conformation of main chain caused by TCB. Likewise, both side chains (I and II) follow the same trend, with slightly higher values of $R_{\mathrm{g}}$ and $d_{\mathrm{t}}$ in TCB, suggesting that alkyl chains are slightly stretched away from the main chain in the presence of TCB. These results indicate that TCB results in a more extended confirmation of D-PDPP4T-HD than CF. Such extended conformation facilitates polymer crystallization via $\pi-\pi$ interaction. This is in excellent agreement with UV-vis-NIR absorption spectra shown in Fig. 1, where the $0-0$ peak of $\beta_{2}$ phase is at longer wavelengths, which is generally associated with a more extended excited state wave function via combination of enhanced $\pi-\pi$ stacking and increased co-planarity of the heteroaromatic units in the main chain.

Polymorphism in the solid state. Interestingly, polymer chains of D-PDPP4T-HD partly resemble their solution polymorph in the solid state. Fig. 4 shows the optical absorption and steadystate PL spectra of D-PDPP4T-HD thin films spin-coated from solutions with various CF:TCB ratios. Small amounts of TCB are able of generating the $\beta_{2}$ phase in the solid state. Only pure CF leads to the formation of a pure $\beta_{1}$ phase. This can be attributed to the much higher boiling point of TCB $\left(214^{\circ} \mathrm{C}\right)$ compared to $\mathrm{CF}\left(61^{\circ} \mathrm{C}\right)$ and the corresponding difference in vapor pressure (197 vs. $0.46 \mathrm{~mm} \mathrm{Hg}$ at $\left.25^{\circ} \mathrm{C}\right)^{39}$. Hence, at the final stage of film formation during spin-coating, a highly concentrated liquid-like film is formed with TCB as the main solvent, since CF has evaporated. At that stage TCB controls the film formation and thus the morphology. Although the emission peak of the $\beta_{1}$ phase is red-shifted from $816 \mathrm{~nm}$ for solutions to around $860 \mathrm{~nm}$ for solid thin films, the emission peak of $\beta_{2}$ phase remains at the same position $(890 \mathrm{~nm})$, as shown in Fig. $4 \mathrm{~b}$. Interestingly, the film with the $\beta_{2}$ polymorph can be transformed back into $\beta_{1}$ by processing CF over the film (Supplementary Fig. 11). As shown in Fig. 4c-e this feature enables patterning of the $\beta_{1}$ and $\beta_{2}$ phase in films, creating a location-dependent functionality. a

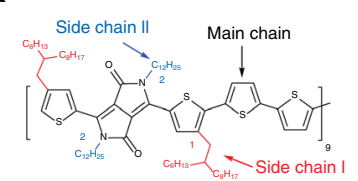

CF

TCB

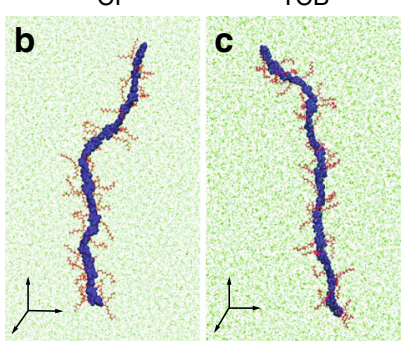

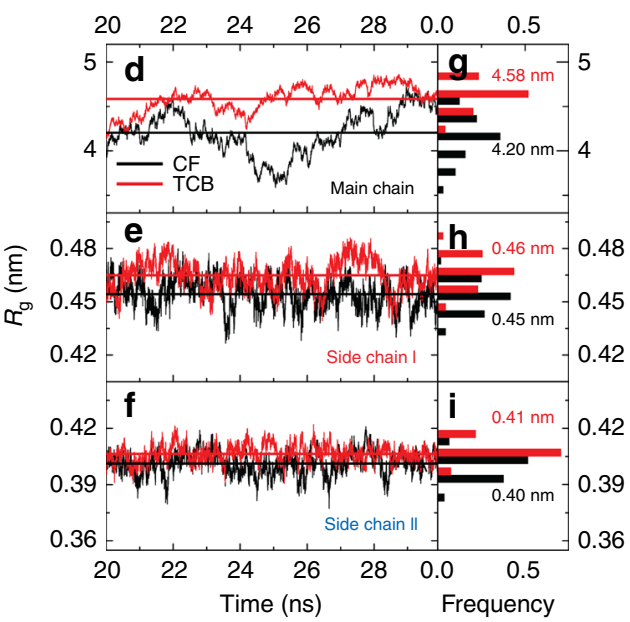

Fig. 3 Molecular dynamics simulations. a Chemical structure for a D-PDPP4T-HD nonamer. b, c Snapshots from trajectories of D-PDPP4T-HD nonamer chains in chloroform (CF) and 1,2,4-trichlorobenzene (TCB). d, e, f, Gyration radius ( $R_{\mathrm{g}}$ ) of main chain, side chain I and side chain II in CF (black) and TCB (red). The straight lines indicate the average values of $R_{\mathrm{g}} . \mathbf{g}, \mathbf{h}, \mathbf{i}$, Corresponding distributions of $\mathbf{d}, \mathbf{e}, \mathbf{f}$. j, $\mathbf{k}, \mathbf{I}$, Head-to-tail distance ( $d_{\mathrm{t}}$ ) of main chain, side chain I and side chain II in CF (black) and TCB (red). The straight lines indicate the average values of $d_{\mathrm{t}}$. $\mathbf{m}, \mathbf{n}, \mathbf{o}$, Corresponding distributions of $\mathbf{j}, \mathbf{k}, \mathbf{I}$. The last 10 ns simulation time after equilibration was selected for sampling. If all chains were straight, the $d_{\mathrm{t}}$ of main chain, side chain I and side chain II would be $18.52,1.62$, and $1.41 \mathrm{~nm}$, respectively 

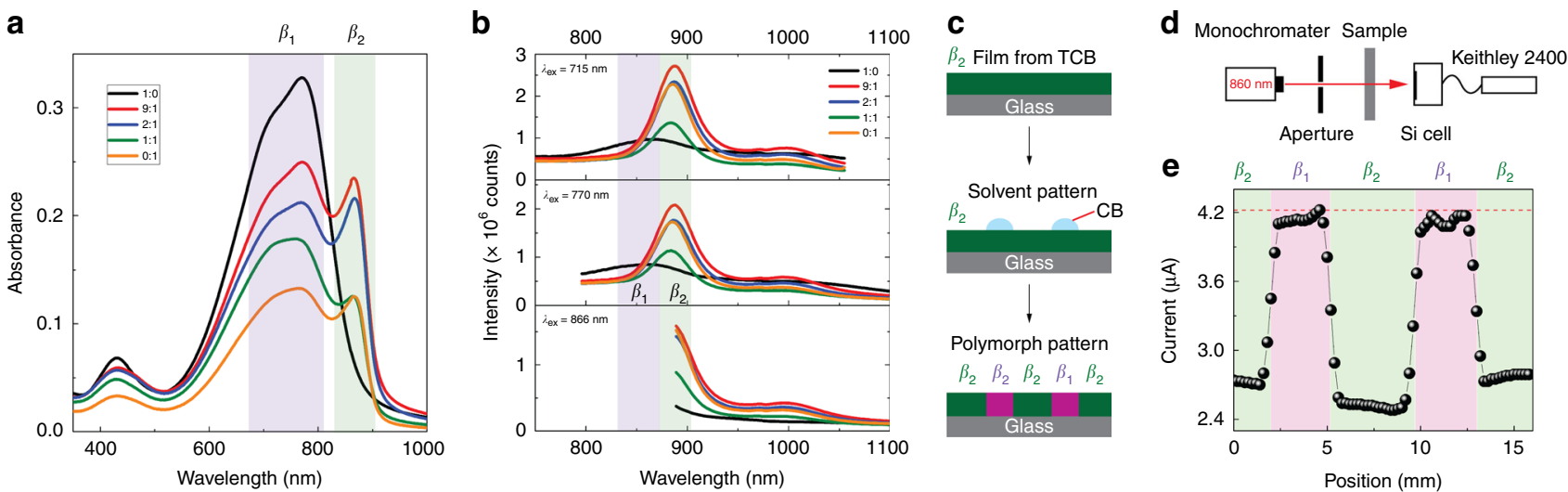

Fig. 4 Optical absorption and steady-state emission spectra of thin films. a UV-vis-NIR absorption of D-PDPP4T-HD thin films spin-coated from chloroform:1,2,4-trichlorobenzene (CF:TCB) solutions with the polymer concentration of $3 \mathrm{mg} \mathrm{mL}-1$. The characteristic peaks for the $\beta_{1}$ and $\beta_{2}$ polymorphs are indicated by purple and green frames, respectively. b Corresponding steady-state PL spectra. c A polymorph pattern is realized by locally exposing a TCB spin-coated ( $\beta_{2}$ phase) film to chlorobenzene (CB). d Schematic illustration of the set up used to measure the polymorph pattern, where 860 nm light with a beam radius of $0.5 \mathrm{~mm}$ is used. e Transmitted light at $860 \mathrm{~nm}$ as a function of position. The red dash line ( $4.22 \mu \mathrm{A})$ indicates the current measured for bare glass without polymer film

It has been reported that long-term solution aging has a pronounced impact on polymer self-assembly in both solution and solid state ${ }^{40}$. Hence, pure TCB solutions of D-PDPP4T-HD were aged from 2 to 8 days. The solution aging results in a significant increase in the intensity of $0-0$ absorption peak of the $\beta_{2}$ phase (Supplementary Fig. 12). The kinetics of aggregate formation from the amorphous $\alpha$ phase demonstrate that the growth rate of the $\beta_{2}$ phase in CF:TCB is three orders of magnitude lower than that of the $\beta_{1}$ phase in CF (Supplementary Fig. 13 and Supplementary Table 4). We find that aged solutions affect the solid state in a different way. By aging pure CF solutions for 2 days, the deposited D-PDPP4T-HD thin film shows a clear shoulder at $870 \mathrm{~nm}$, indicating formation of some $\beta_{2}$ phase (Supplementary Fig. 14). On the other hand, aging a CF solution containing TCB has no effect on the thin film UV-vis-NIR spectrum (Supplementary Fig. 15). The difference in aging behavior between solution and film is not fully understood yet, but it suggests that when some $\beta_{2}$ phase is present in solution, it acts as a seed for the film morphology.

Grazing incidence wide-angle X-ray scattering (GIWAXS) is employed to gain structural information of the two aggregated phases including in-plane organization and orientational texture. In order to increase the scattering intensity, drop-casting is utilized to deposit thin films. We emphasize that drop-cast films depict identical polymer aggregation characteristics in the UVvis-NIR spectra as spin-coated ones (Fig. 4 and Supplementary Fig. 16). All drop-cast films exhibit similar surface topographies with granular features, determined by tapping-mode atomic force microscopy (AFM, Supplementary Fig. 17). The GIWAXS 1D profiles are shown in Fig. 5a, b. In the in-plane profiles, strong lamellar (100), (200), and even (300) peaks are evident for all samples. In the out-of-plane profiles, the $(010)$ peak is seen for all samples. These results demonstrate that D-PDPP4T-HD is selforganized with a predominant face-on packing on the surface, independent of the CF:TCB ratio. Quantitative analysis is performed by assessing the lamellar spacing $\left(d_{\text {lam }}\right)$ and $\pi$-stacking distance $\left(d_{\pi}\right)$, as shown in Fig. $5 c$. All films exhibit almost identical $d_{\text {lam }}$ values between 2.34 and $2.38 \mathrm{~nm}$. On the contrary, the film processed from pristine CF (1:0) displays slightly larger $d_{\pi}(0.39 \mathrm{~nm})$ compared to the other films $(\sim 0.37$ $\mathrm{nm})$. This can be attributed to the less extended conformation of the main chain in pristine CF, as simulated in Fig. 3. Moreover, in the presence of TCB, $d_{\pi}$ remains almost unchanged with TCB content. We conclude that both $\beta_{1}$ and $\beta_{2}$ phases have face-on packing orientation, and their $d_{\pi} \mathrm{s}$ are slightly different. Another difference is shown in the orientational distribution. The (100) interlayer reflection intensity profile is plotted as pole figure for the azimuthal angle between $-90^{\circ}$ and $90^{\circ}$. From this plot it is possible to determine the proportion of the face-on orientation (Supplementary Fig. 18). The trend of $\gamma_{\text {face }}$ reveals a slightly higher face-on population in the $\beta_{1}$ phase than in the $\beta_{2}$ phase.

Thermal properties of two different aggregated phases of DPDPP4T-HD are investigated by differential scanning calorimetry (DSC). First, the polymer precipitated from CF in methanol, defined as the standard sample, is measured as control with scan rates of 10 and $40{ }^{\circ} \mathrm{C}$ min $^{-1}$ (Fig. 6a and Supplementary Fig. 19). In the heating cycle, a melting temperature $\left(T_{\mathrm{m}}\right)$ of $249.6^{\circ} \mathrm{C}$ is found; in the cooling process, two close recrystallization peaks at 236.2 and $233.5^{\circ} \mathrm{C}$ are observed (Fig. 6a). To measure DSC of the $\beta_{1}$ and $\beta_{2}$ aggregated phases, a simple method is used to process the polymer from the different solvents (Supplementary Fig. 20). Briefly, the inside and bottom walls of a 10 -mL glass vial are first coated with poly(acrylic acid) (PAA). After the PAA solidification, a concentrated solution of D-PDPP4T-HD with various solvents is poured into the coated vial, and then dried under vacuum. Consequently, the free-standing D-PDPP4T-HD is obtained by dissolving PAA with Milli-Q water. On the basis of this approach, DSC samples with $\beta_{1}$ or $\beta_{2}$ phases are fabricated for CF:TCB 1:0 or 9:1, and the first heating cycle in the DSC is recorded with slower scanning rate $\left(2{ }^{\circ} \mathrm{C} \mathrm{min}-1\right)$, as shown in Fig. 6b. In comparison to the standard sample, both $\beta_{1}$ and $\beta_{2}$ phases exhibit a cold crystallization transition $\left(T_{\mathrm{c}}\right)$ with peak positions at 228.5 and $225.9^{\circ} \mathrm{C}$. For this $T_{\mathrm{c}}$ process, the enthalpy of $\beta_{2}$ is $40 \%$ higher than that of $\beta_{1}$. Note that both aggregated phases display a similar $T_{\mathrm{m}}$ of $\sim 242^{\circ} \mathrm{C}$, which is $8{ }^{\circ} \mathrm{C}$ lower compared to the precipitated polymer.

Further investigation on thermal properties of the two aggregated phases is conducted through in situ UV-vis-NIR spectroscopy by heating/cooling D-PDPP4T-HD thin films under nitrogen atmosphere. The heating and cooling rates are 2 and $1{ }^{\circ} \mathrm{C} \min ^{-1}$, respectively, and the maximum heating temperature reaches $270^{\circ} \mathrm{C}$. Upon annealing, the $0-0$ peak and $0-1$ vibronic shoulders of the $\beta_{1}$ phase blue shift and their intensities gradually drop (Fig. $6 \mathrm{c}$ ). At $250^{\circ} \mathrm{C}\left(>T_{\mathrm{m}}\right)$, these two signals are replaced by one new main peak. At $270^{\circ} \mathrm{C}$, this new peak is shifted to around $645 \mathrm{~nm}$, resembling the $\alpha$ phase in 

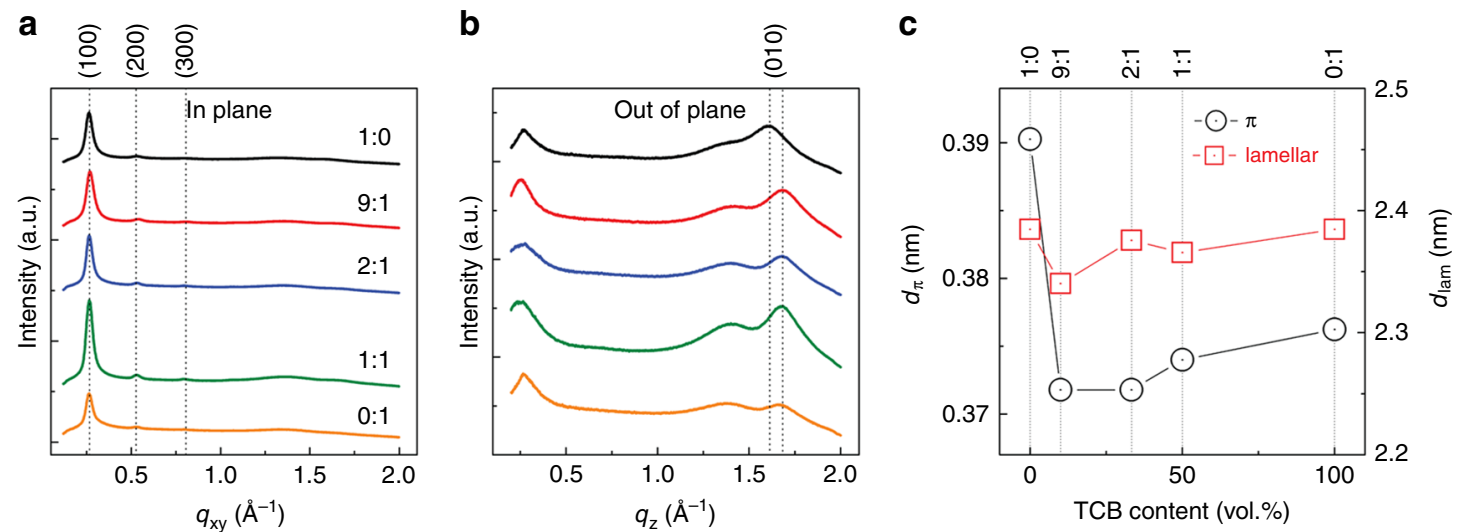

Fig. 5 Grazing incidence wide-angle X-ray scattering (GIWAXS) of thin films. GIWAXS of D-PDPP4T-HD thin films cast from different chloroform:1,2,4trichlorobenzene (CF:TCB) solutions. a In-plane profile. b Out-of-plane profile. c Lamellar spacing $\left(d_{\text {lam }}\right)$ and $\pi$-stacking distance $\left(d_{\pi}\right)$. Note that the scale in panel $\mathbf{c}$ is different for $d_{\pi}$ and $d_{\text {lam }}$
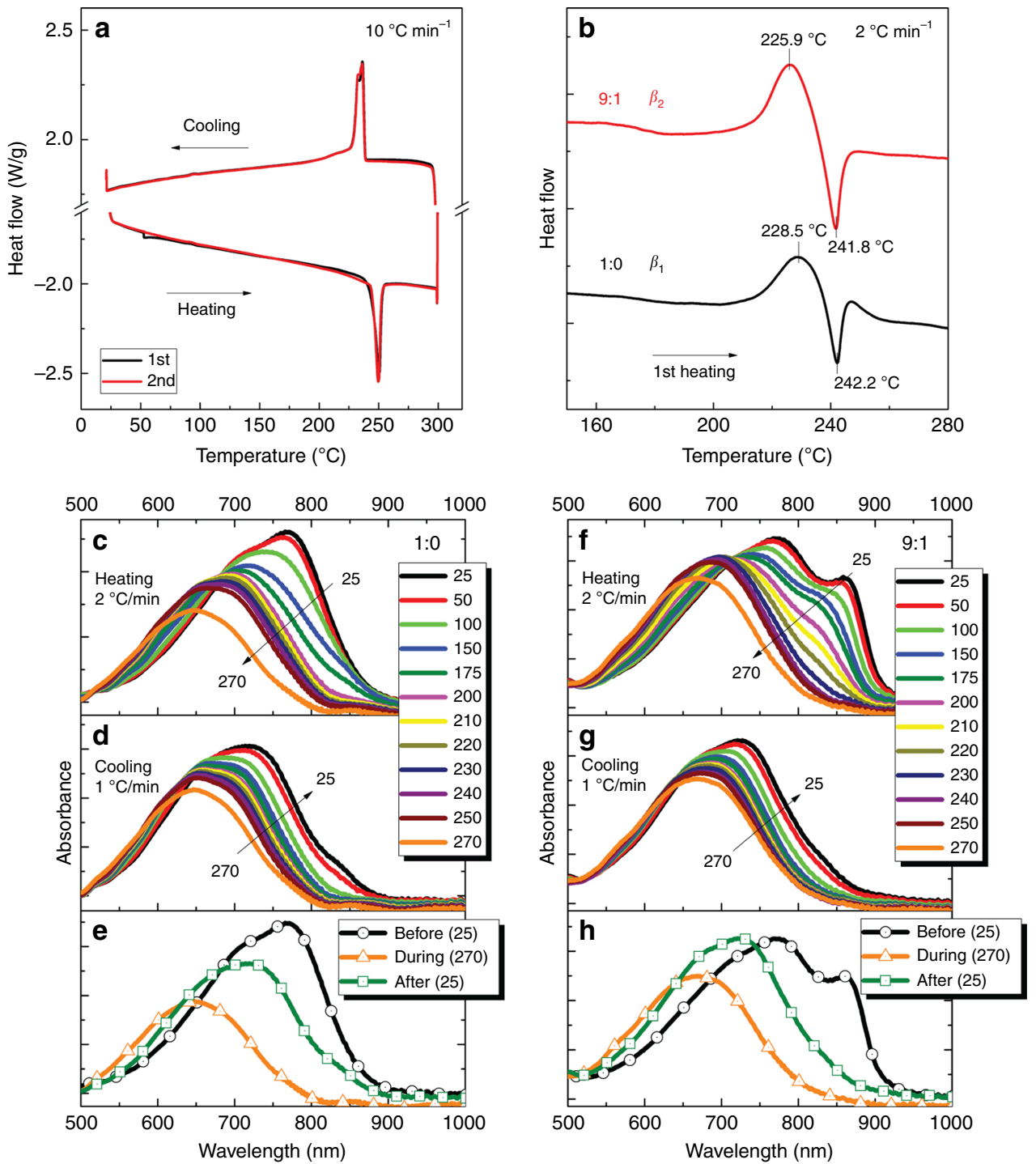

Fig. 6 Thermal behavior of polymorphs. a Standard DSC thermograms of D-PDPP4T-HD precipitated from chloroform in methanol. The scanning rate is $10^{\circ} \mathrm{C} \mathrm{min}-1$. b The first heating DSC thermogram for D-PDPP4T-HD re-dissolved and dried from different solvents. The scanning rate is $2^{\circ} \mathrm{C}$ min ${ }^{-1}$. $\mathbf{c}, \mathbf{d}, \mathbf{e}$, $\mathbf{f}, \mathbf{g}$, h, In situ optical absorption during heating and cooling of D-PDPP4T-HD thin films spin-coated from CF:TCB 1:0 (c, d, e) and 9:1 (f, $\mathbf{g}$, h). N 2 atmosphere is utilized to protect the polymer films. The heating and cooling rates are $2^{\circ} \mathrm{C} \mathrm{min}^{-1}$ and $1^{\circ} \mathrm{C} \mathrm{min}^{-1}$, respectively. The bottom panels compare the optical absorption before and after thermal annealing 
solution (Fig. 1a and Supplementary Fig. 2). The cooling process results in a red shift of absorption spectra and the re-appearance of the $0-0$ and $0-1$ vibronic features at $720 \mathrm{~nm}$ and $660 \mathrm{~nm}$ (Fig. 6d). A direct comparison of the absorption spectra before and after annealing is shown in Fig. 6e and reveals that thermal cycling from $\beta_{1} \rightarrow \alpha \rightarrow \beta_{1}$ is partially reversible during the time scale of the experiment, but ultimately results in a $\sim 50-\mathrm{nm}$ blue shift. Interestingly, a tiny fraction of the $\beta_{2}$ phase is generated at around $850 \mathrm{~nm}$ during crystallization. Compared to the $\beta_{1}$ phase, the thermal cycling of the $\beta_{2}$ phase exhibits a substantially lower reversibility. The absorbance at $870 \mathrm{~nm}$ vanishes upon heating and is only partly restored in the cooling cycle, as shown in Fig. 6f, g, h and Supplementary Fig. 21. We find that faster heating/cooling rates result in more recovery of the $\beta_{2}$ phase (Supplementary Figs. 21 and 22). If the $\beta_{1}$ and $\beta_{2}$ phases are heated to a fully disordered $\alpha$ phase upon annealing to $270{ }^{\circ} \mathrm{C}$, the two samples are expected to form the same state upon returning to $25^{\circ} \mathrm{C}$, because the starting point at $270{ }^{\circ} \mathrm{C}$ is the same. This is exactly what is observed, especially for the slower heating and cooling experiment in which the sample is longer above the melt transition where the chains lose their ordered structure. The result that an ordered phase only partly reappears upon cooling from the melt can be attributed to the fact that the formation of a semi-crystalline phase requires polymer chains to realign from the disordered melt. This is hampered by the reduced mobility of polymer chains in the solid films when the temperature drops below the crystallization temperature $\left(233-236^{\circ} \mathrm{C}\right.$, Fig. 6a). The fact that cooling from the melt produces more $\beta_{1}$ phase than $\beta_{2}$ phase is likely a kinetic effect. In solution the $\beta_{2}$ phase forms also more slowly (Supplementary Fig. 13). It is also consistent with the idea that $\beta_{2}$ is the more ordered phase (because it is a lower optical band gap).

Effect of polymorphism in transistors and solar cells. To investigate the impact of polymorphism of D-PDPP4T-HD on the charge transport in electronic devices field-effect transistors (FETs) were used with a bottom-contact top-gate architecture. The FETs were fabricated on silicon wafers with thermally grown $\mathrm{SiO}_{2}$, a spin-coated D-PDPP4T-HD layer from mixed CF:TCB solutions, a CYTOP insulator layer, and a silver gate contact. To remove residual solvent, especially $\mathrm{TCB}$, all polymer films are annealed at $100{ }^{\circ} \mathrm{C}$ for $0.5 \mathrm{~h}$ in a nitrogen glovebox, which has little effect on film morphology and molecular ordering of conjugated polymers (Fig. 6) ${ }^{41,42}$. Fig. 7 a exhibits the transfer characteristics of both aggregated phases at a drain voltage $\left(V_{\mathrm{DS}}\right)$ of $-30 \mathrm{~V}$. The drain current $\left(I_{\mathrm{DS}}\right)$ of the $\beta_{1}$ phase (from CF:TCB 1:0) is lower than that of $\beta_{2}$ phase (from CF:TCB 2:1) and the saturated hole mobility $\left(\mu_{\mathrm{h}}\right)$ is $0.058 \pm 0.014 \mathrm{~cm}^{2} \mathrm{~V}^{-1} \mathrm{~s}^{-1}$ for $\beta_{1}$ and $0.26 \pm 0.11 \mathrm{~cm}^{2} \mathrm{~V}^{-1} \mathrm{~s}^{-1}$ for $\beta_{2}$, when averaged over six devices. The on/off ratio $\left(I_{\text {on }} / I_{\text {off }}\right)$ of about $10^{3}$ and threshold voltage $\left(V_{\mathrm{T}}\right)$ of circa $+3 \mathrm{~V}$ are similar for the $\beta_{1}$ and $\beta_{2}$ phases. Fig. $7 \mathrm{~b}$ shows the effect of the CF:TCB ratio in the solution used to cast the film on the hole mobility. When this ratio exceeds 2:1, the charge transport remains almost unchanged with a mobility on the order of $0.25 \mathrm{~cm}^{2} \mathrm{~V}^{-1} \mathrm{~s}^{-1}$. Compared to $\beta_{1}$, this 4 -fold higher mobility of the $\beta_{2}$ phase is consistent with its reduced $\pi$ stacking distance as inferred from GIWAXS and the longer conjugation length as inferred from UV-vis-NIR absorption spectroscopy. ${ }^{43,44}$

It is also of interest to evaluate if the two semi-crystalline polymorphs $\beta_{1}$ and $\beta_{2}$ can be identified in donor-acceptor bulkheterojunction organic photovoltaic (OPV) cells with DPDPP4T-HD as the donor polymer and $[6,6]$-phenyl- $\mathrm{C}_{71}$-butyric acid methyl ester ([70]PCBM) as acceptor material. To study this, conventional configuration (ITO/PEDOT:PSS/D-PDPP4T-HD: [70]PCBM/LiF/Al) solar cells are fabricated. $\beta_{1}$-only devices fabricated using pure CF afford a very low short-circuit current density $\left(J_{\mathrm{sc}}\right.$, integrated from the external quantum efficiency (EQE) with AM1.5 G solar spectrum) of $2.6 \mathrm{~mA} \mathrm{~cm}^{-2}$ and a power conversion efficiency (PCE) of only $1.2 \%$, due to a coarse phase separation between D-PDPP4T-HD and [70]PCBM caused by spinodal liquid-liquid decomposition during drying 45 (Fig. 7c, f, g and Supplementary Table 5). Using 1,2-dichlorobenzene (o-DCB) as cosolvent in $\mathrm{CF}$, it is possible to retain a $\beta_{1}$-only polymorph, but $o$-DCB is not capable of eliminating the spinodal phase separation in the D-PDPP4T-HD:[70]PCBM blend and the PCE remains low (Supplementary Figs. 23 and 24). In solar cells processed with TCB as cosolvent spinodal demixing is fully suppressed and the $\beta_{2}$ phase is present. After optimization, the TCB concentration for the best device is $8 \%$ (Supplementary Fig. 25). As shown in Fig. 7f, the current density of solar cells processed from CF with $8 \%$ TCB is almost 5-fold higher than that processed from neat $\mathrm{CF}$, resulting in a significant improvement in PCE from $1.2 \%$ to $6.4 \%$. Transmission electron microscopy (TEM) images reveal the fibrillar microstructure (Fig. 7d), which facilitates exciton diffusion and charge separation. Furthermore, the EQE in the wavelength range from 820 to $900 \mathrm{~nm}$ reaches 0.4 , which originates from $\beta_{2}$ phase (Fig. $7 \mathrm{~g}$ ). Diphenyl ether (DPE) as co-solvent ( $2 \%$ in CF) is also capable of suppressing coarse phase separation and generating the $\beta_{2}$ phase, but the EQE at $860 \mathrm{~nm}\left(\mathrm{EQE}_{860}\right)$ is only half of the TCB $(8 \%$ in CF) sample, as shown in Fig. $7 \mathrm{~g}$ and Table 1 . The resultant $2 \%$ DPE device provides a PCE of $6.2 \%$, in agreement with our previous report ${ }^{31}$. This confirms that the $\beta_{2}$ phase has a significant contribution to the OPV performance. The somewhat higher $V_{\text {oc }}$ found for $\beta_{1}$-phase solar cells compared to $\beta_{2}$-phase devices $(0.66$ vs. $0.62 \mathrm{~V})$, is consistent with the higher ionization potential for $\beta_{1}(I P=4.88 \mathrm{eV})$ than for $\beta_{2}(I P=4.80 \mathrm{eV})$ inferred from ultraviolet photoemission spectroscopy (UPS) (Supplementary Fig. 26).

Two other well-known cosolvents for DPP polymers, 1,8diiodooctane (DIO) and 1-chloronaphthalene $(\mathrm{CN})$, are also tested for polymorph control. In both cases, the EQE values in the 820 to $900 \mathrm{~nm}$ spectral range are relatively pronounced, suggesting the presence of $\beta_{2}$ phase (Supplementary Fig. 27). More interestingly, by comparing all five different cosolvents, it seems that the fibrillar microstructure and the $\beta_{2}$ phase appear in the photoactive layer simultaneously, resulting in improved device performance (Supplementary Figs. 24 and 27).

Generality and mechanism of polymorphism. We are in the process of further establishing the structural features of the polymer that enable the $\beta_{1}$ and $\beta_{2}$ polymorphism by varying type and length of the side chains. At present we are looking further into which combination of side chains (linear, branched, branching position, length, number and attachment position of side chains) for the PDPP4T backbone provides a similar polymorphism. Preliminary results identify the $\beta_{1}-\beta_{2}$ polymorphism in three other DPP polymers for different linear side chains on the DPP core (Supplementary Fig. 28). However, we have not been able to achieve a similar solvent induced $\beta_{1} \rightarrow \alpha \rightarrow \beta_{2}$ transition for a PDPP4T polymer with branched 2-ethylhexyl side chains on the DPP unit and on the two adjacent thiophene units, or for a PDPP4T derivative that has 2-decyltetradecyl side chains on the DPP unit and an unsubstituted $4 \mathrm{~T}$ segment. This demonstrates that the substitution effects are subtle and that establishing structural-property relations requires further investigation. Without doubt solvent quality plays a critical role in chain conformation of conjugated polymers ${ }^{46,47}$, and the appearance of the $\beta_{2}$ phase in this study is associated with the balance between molecule-molecule and molecule-solvent interactions. We have tested if the Hansen solubility parameters, which 
a

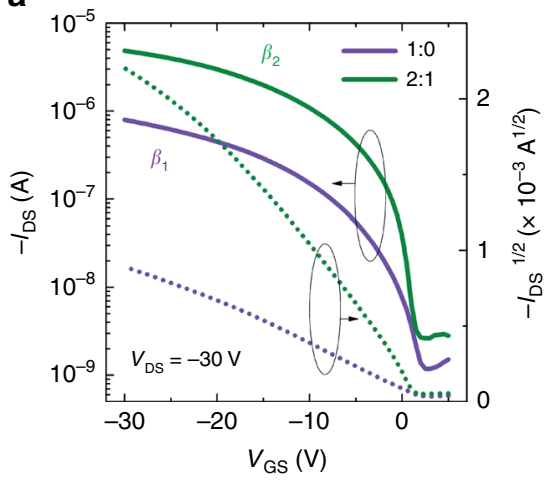

b

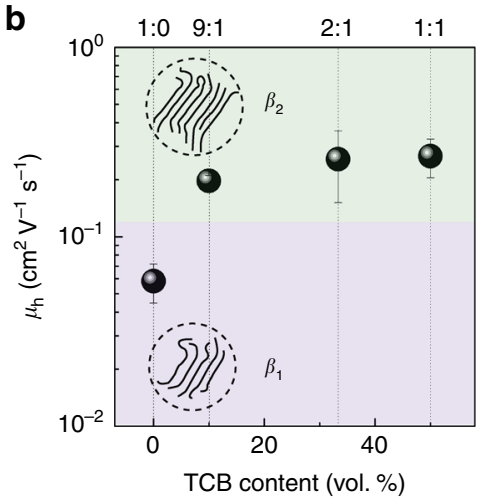

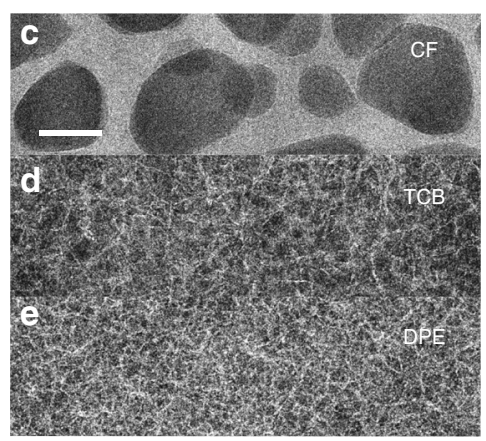
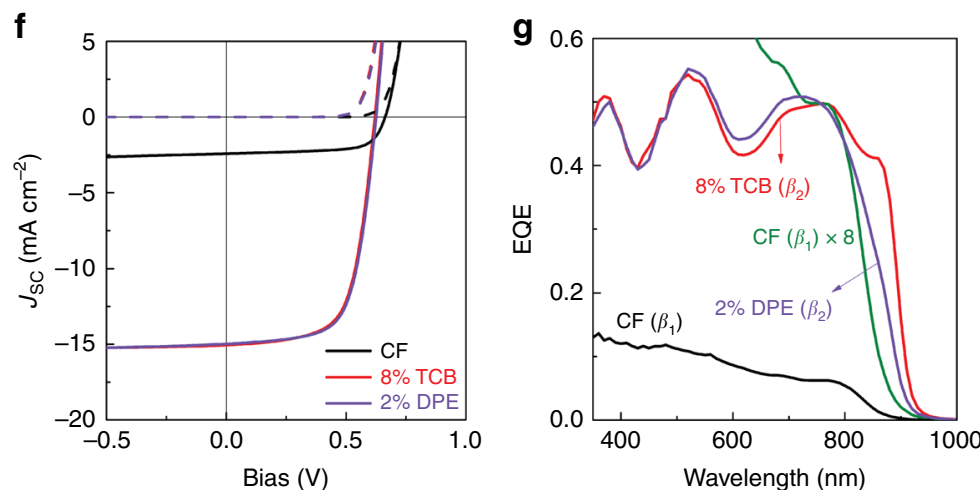

Fig. 7 Field-effect transistors and solar cells of the two polymorphs. a Transfer characteristics of D-PDPP4T-HD field-effect transistors for the $\beta_{1}$ and $\beta_{2}$ polymorphs processed from chloroform:1,2,4-trichlorobenzene (CF:TCB) solutions (1:0) and (2:1). b Average (6 devices) field-effect mobility as a function of TCB content. Error bars are standard deviation. $\mathbf{c}, \mathbf{d}$, e TEM images of D-PDPP4T-HD:[70]PCBM blends with various cosolvents. All images share the same scale bar of $200 \mathrm{~nm}$. $\mathbf{f} J-V$ characteristics of D-PDPP4T-HD:[70]PCBM solar cells under simulated AM1.5 G (100 mW cm-2) illumination (solid lines). The dash lines indicate the dark current. $\mathbf{g}$ Corresponding EQE spectra

describe the dispersive $\left(\delta_{\mathrm{D}}\right)$, polar $\left(\delta_{\mathrm{P}}\right)$ and hydrogen-bonding $\left(\delta_{\mathrm{H}}\right)$ forces between polymers and solvent molecules, can provide an interpretation for the solvent-related polymorphism ${ }^{48,49}$. Besides $\mathrm{CF}$ and $\mathrm{TCB}$, we also conducted absorption measurements for DPDPP4T-HD in 1,2-dichlorobenzene, 1-chloronaphthalene and 1,1,2,2-tetrachloroethane (TCE), benzal chloride and (2-chloroethyl)benzene (Supplementary Fig. 29 and Supplementary Table 6). The results show that for several solvents with Hansen parameters that are similar to those of TCB, $\beta_{2}$ formation is not commonly observed in solution, while it is observed in TCE which has parameters closer to CF. Clearly, further efforts are required to understand the mechanism and the origin of polymorphism in conjugated polymers.

\section{Discussion}

Polymorphism of the aggregated phases of a low-bandgap conjugated polymer, namely D-PDPP4T-HD, is identified in solution and in the solid state. The utilization of TCB as solvent or cosolvent results in the formation of a second aggregated phase $\left(\beta_{2}\right)$ with a distinct absorption peak at around $870 \mathrm{~nm}$. Luminescence lifetimes of $29 \mathrm{ps}, 78 \mathrm{ps}$, and $129 \mathrm{ps}$ are determined by timeresolved PL spectroscopy for $\alpha, \beta_{1}$, and $\beta_{2}$, respectively. The PL quantum yield of the $\beta_{2}$ phase is more than 30 -fold higher than that of $\beta_{1}$. In the solid state, the aggregated phase, observed from solution, is retained. The spectroscopic, structural, thermal properties and electronic device performance of thin films with two different aggregated phases have been explored, as summarized in Table 1. (1) Compared to $\beta_{1}, \beta_{2}$ has a new 0-0 absorption peak at $870 \mathrm{~nm}$, accompanied by an additional emission peak at $890 \mathrm{~nm}$. The corresponding optical bandgap is decreased to $1.37 \mathrm{eV}$. (2) Both aggregated phases possess similar microstructures and polymer packing. (3) Cold crystallization is observed for both $\beta_{1}$ and $\beta_{2}$, but with larger enthalpy for $\beta_{2}$. (4) $\beta_{2}$ exhibits higher field-effect mobility in OFETs than $\beta_{1}$, which can be attributed to the reduced $d_{\pi}$. More importantly, the additional absorption of $\beta_{2}$ in the near-infrared region enhances the photovoltaic performance of polymer solar cells.

The strategy for polymorphism control reported in this study is compatible with automated and large scale deposition methods such as ink-printing and roll-to-roll techniques. Interestingly, the results indicate that programmable inks with only one polymer can be designed to pattern different polymorphs for electronic devices, creating location-dependent functionalities. On the other hand, D-PDPP4T-HD can be a good candidate for switchable photodetectors and/or phototransistors in the near-infrared region, due to its tunable ability of light absorption. These results offer us a further understanding of the role of polymer polymorphism in structure-property relations in plastic electronics.

\section{Methods}

Materials. D-PDPP4T-HD was synthesized according to the literature procedure ${ }^{31}$. In this study, two batches of polymers are used, batch I $\left(M_{\mathrm{n}}=71.5 \mathrm{~kg} \mathrm{~mol}^{-1}\right.$ and $\left.M_{\mathrm{w}} / M_{\mathrm{n}}=2.5\right)$ and batch II $\left(M_{\mathrm{n}}=63.4 \mathrm{~kg} \mathrm{~mol}^{-1}\right.$ and $\left.M_{\mathrm{w}} / M_{\mathrm{n}}=2.0\right)$, as determined by gel permeation chromatography (GPC) at $140^{\circ} \mathrm{C}$ on a PL-GPC 120 system using a PL-GEL $10 \mathrm{~mm}$ MIXED-C column with 1,2-dichlorobenzene (o-DCB) as the eluent and polystyrene as internal standards. Before GPC measurement, polymer was dissolved in $o$-DCB at $140^{\circ} \mathrm{C}$ for $1 \mathrm{~h}$. Such minor difference in molecular weight has no effect on the polymorphism of D-PDPP4T-HD, as shown in Supplementary Fig. 30. Batch I is the one used in our previous report ${ }^{31}$. In this study, batch I is used for GIWAXS samples, and batch II for the other experiments and measurements. The similar performance is also apparent from the reproducibility of the photovoltaic performance compared to the previous study. 


\section{Table 1 Optical, structural, thermal, and electrical properties of the two polymorphs}

\begin{tabular}{|c|c|c|c|}
\hline Property & & $\beta_{1}$ & $\beta_{2}$ \\
\hline \multirow[t]{2}{*}{$\lambda_{\mathrm{O}-\mathrm{O}}(\mathrm{nm})$} & Absorptiona & 770 & 870 \\
\hline & $\mathrm{PL}^{\mathrm{b}}$ & 860 & 890 \\
\hline$E_{g}{ }^{c}(e V)$ & & 1.42 & 1.37 \\
\hline IP $(e V)^{d}$ & & 4.88 & 4.80 \\
\hline \multirow[t]{2}{*}{$d(\mathrm{~nm})$} & $d_{\pi}{ }^{e}$ & 0.39 & 0.37 \\
\hline & $d_{\text {lam }}^{f}$ & 2.4 & 2.4 \\
\hline $\begin{array}{l}\text { Polymer orientation } \\
\left(\gamma_{\text {face }} g, \%\right)\end{array}$ & & 77 & 74 \\
\hline \multirow[t]{2}{*}{$T\left({ }^{\circ} \mathrm{C}\right)$} & $T_{\mathrm{c}}^{\mathrm{h}}$ & 228.5 & 225.9 \\
\hline & $T_{\mathrm{m}}^{\mathrm{i}}$ & 242.2 & 241.8 \\
\hline Enthalpy ${ }^{j}\left(\mathrm{~J} \mathrm{~g}^{-1}\right)$ & & 22.3 & 31.4 \\
\hline$\mu_{\mathrm{FET}}{ }^{\mathrm{k}}\left(\mathrm{cm}^{2} \mathrm{~V}^{-1} \mathrm{~s}^{-1}\right)$ & & $0.058 \pm 0.014$ & $0.26 \pm 0.11$ \\
\hline \multirow[t]{3}{*}{$V_{\text {oc }}$ I $(V)$} & & 0.66 & 0.62 \\
\hline & & $(0.663 \pm$ & $(0.621 \pm$ \\
\hline & & $0.000)$ & $0.002)$ \\
\hline \multirow[t]{2}{*}{$J_{\mathrm{sc}, \mathrm{sr}} \mathrm{m}\left(\mathrm{mA} \mathrm{cm} \mathrm{m}^{-2}\right)$} & & 2.6 & 15.5 \\
\hline & & $(2.5 \pm 0.1)$ & $(15.1 \pm 0.5)$ \\
\hline \multirow[t]{2}{*}{$\operatorname{PCE}^{\mathrm{n}}(\%)$} & & 1.2 & 6.4 \\
\hline & & $(1.1 \pm 0.0)$ & $(6.2 \pm 0.1)$ \\
\hline $\mathrm{EQE}_{860^{\circ}}$ & & 0.01 & 0.41 \\
\hline
\end{tabular}

aUV-vis-NIR absorption

bPhotoluminescence

cOptical bandgap

donization potential from UPS

$\mathrm{e}_{\pi-\pi}$ stacking distance

fLamellar spacing

gPercentage of face-on orientation

hCold crystallization temperature

iMelting point

Values are for cold crystallization process and obtained from Fig. $6 \mathrm{~b}$

kHole mobility based on the field-effect measurement

IOpen-circuit voltage, average and standard deviation from five cells between parenthesis

mShort-circuit current density determined by integrating the EQE spectrum with AM $1.5 \mathrm{G}$

spectrum

Power conversion efficiency

${ }^{\circ} \mathrm{EQE}$ at $860 \mathrm{~nm}$

Optical spectroscopy. UV-vis-NIR absorption spectroscopy was conducted on a PerkinElmer Lambda 1050 spectrophotometer at various temperatures for both solution and solid states. Steady-state photoluminescence spectra were recorded though an Edinburgh Instruments FLSP920 double-monochromator luminescence spectrometer equipped with a nitrogen-cooled near-IR sensitive photomultiplier (Hamamatsu)

Time-resolved photoluminescence. For time-resolved photoluminescence measurements the samples were excited with the wavelength-tunable output of an Optical Parametric Oscillator (Radiantis Inspire HF-100), itself pumped by the fundamental at $820 \mathrm{~nm}$ of a Ti:sapphire fs oscillator (Spectra Physics MaiTai eHP), yielding $100 \mathrm{fs}$ pulses at a repetition rate of $80 \mathrm{MHz}$. The PL of the samples was collected with an optical telescope consisting of two plano-convex lenses matched in focal length to a spectrograph (PI Spectra Pro SP2300) for wavelength dispersion and detected with a streak camera (Hamamatsu C10910) system with a temporal resolution of $1.4 \mathrm{ps}$. The PL data was acquired in photon counting mode using the streak camera software (HPDTA) and exported to Origin Pro 2017 for further analysis.

Spectral weight analysis. To separate the absorbance spectra and determine the spectral weight of each polymorph phase, MCR-ALS analysis ${ }^{46,47}$ was applied to an augmented matrix comprised of the absorption spectra of each sample (solvent ratio). Singular value decomposition (SVD) was applied to estimate the number of components present in the data matrix. Several constraints were applied to the analysis such as non-negativity of absorbance and concentration. Furthermore, the spectrum of one component was fixed to the absorption spectrum of $\alpha$ (in TCB at $80^{\circ} \mathrm{C}$ ), while the input spectra of the other two components were kept unknown $(\mathrm{NaN})$. Finally, the input concentration of $\beta_{1}$ was fixed to zero for the 0:1 sample only, while the concentrations of all other data points were kept unknown $(\mathrm{NaN})$. This constraint was applied, as otherwise MCR-ALS outputs the concentration of $\alpha$ to zero for the $0: 1$ sample and overestimates the contribution of $\beta_{1}$, while experimentally emission of $\alpha$ in the 0:1 sample is clearly seen from TRPL, indicating the presence of $\alpha$ in the sample.
Computational methodology. To illustrate the influence of solvents on the polymer conformations, all-atom molecular dynamics (MD) simulations were performed by Gromacs-4.6.7 with a modified general AMBER force field (GAFF) ${ }^{48}$ The atomic charges were re-optimized from the restrained electrostatic potentials (RESP) at the HF/6-31 G** level of theory. The torsion potential parameters for the dihedral angles between the conjugated units (i.e., DPP and thiophene moieties) were derived from density functional theory (DFT) calculations at the $\omega \mathrm{B} 97 \mathrm{XD} / 6$ $31 \mathrm{G}^{* *}$ level. A cutoff of $1.2 \mathrm{~nm}$ was used for all non-bonded interactions. All the MD simulations were carried out under the NPT ensemble at the room temperature and atmosphere pressure with three-dimensional periodic boundary conditions. The Berendsen weak coupling and velocity rescaling thermostat were used to control the pressure and temperature with a coupling time of 1 ps and 0.1 ps, respectively. ${ }^{49,50}$ To construct the polymer solution, a single polymer chain with nine repeat units was first put in a box with the lattice length of $35 \mathrm{~nm}$, then the rest space of the box was filled by solvent molecules. After equilibration, the box length was decreased to $23.04 \mathrm{~nm}$ and $25.75 \mathrm{~nm}$ for the CF and TCB solutions, respectively. A total simulation time of $30 \mathrm{~ns}$ was run and the sampling data were collected every 2 ps from the last balanced time of $10 \mathrm{~ns}$.

Structural characterizations. Grazing incidence wide-angle X-ray scattering (GIWAXS) was performed under vacuum $(\sim 1 \mathrm{mbar})$ at the DELTA Synchrotron using beamline BL09 with a photon energy of $10 \mathrm{keV}$. The incident angle of X-ray beam is around $0.1^{\circ}$, above the critical angle of the polymer film but below that of the substrate. The scattering intensity was detected on a $2 \mathrm{D}$ image plate (MAR$345)$, and the software WAXStools was utilized for data processing and analysis ${ }^{51}$.

Thermal properties. Differential scanning calorimetry (DSC) was determined using a TA Instruments Q2000 DSC in Tzero hermetic pans. Sample preparation from different solvents is described in Supplementary Fig. 20. In situ absorption measurements were performed by fixing a Linkam heating stage into the PerkinElmer Lambda 1050 spectrophotometer with a home-made holder. During the heating/cooling procedures, the samples were protected under nitrogen atmosphere.

Device fabrication and characterization. A bottom-contact top-gate architecture was utilized for the fabrication of OFET devices. The source and drain electrodes with $60 \mathrm{~nm}$ in thickness were deposited by Au evaporation, and the ratio of channel length to width is $1 / 20$. CYTOP insulator was spin-coated on top of D-PDPP4T$\mathrm{HD}$ thin film as dielectric layer, followed by annealing at $100{ }^{\circ} \mathrm{C}$ for $1 \mathrm{~h}$. Finally, 50 $\mathrm{nm} \mathrm{Ag}$ was evaporated as gate electrode. A Keithley 4200-SCS was used for all standard OFET measurement under vacuum.

Pre-patterned indium tin oxide (ITO) substrates ( $15 \Omega$ per square, Naranjo Substrates) were used for photovoltaic devices with a conventional configuration (ITO/PEDOT:PSS/D-PDPP4T-HD:[70]PCBM/LiF/Al). All photoactive layers were deposited by spin-coating from a chloroform solution with different co-solvents. The active area of the cells was 0.09 or $0.16 \mathrm{~cm}^{2}$ as determined by the overlap of the ITO bottom contact and aluminum top contact, and no dependence of photovoltaic performance on the active area was found. The white light $(\sim 100 \mathrm{~mW}$ $\mathrm{cm}^{-2}$ ) from a tungsten-halogen lamp filtered by a Schott GG385 UV filter and a Hoya LB120 daylight filter was used for illumination. $J-V$ characteristics were measured in a $\mathrm{N}_{2}$-filled glove box $\left(<1\right.$ ppm $\mathrm{O}_{2},<1$ ppm $\left.\mathrm{H}_{2} \mathrm{O}\right)$ at ambient temperature, without any preconditioning, using a Keithley 2400 source meter from +2 to $-2 \mathrm{~V}$ in $0.01 \mathrm{~V}$ step size at a rate of $30 \mathrm{~ms}$ per data point. Short-circuit currents under AM1.5 G conditions were estimated from the EQE and integration with the AM1.5 $\mathrm{G}\left(100 \mathrm{~mW} \mathrm{~cm}^{-2}\right)$ solar spectrum. The EQE was recorded by a lock-in amplifier (Stanford Research Systems SR 830) under simulated 1 sun operation conditions with bias light from a $530 \mathrm{~nm}$ high-power LEDs (Thorlabs). As probe light, the light from a $50 \mathrm{~W}$ tungsten halogen lamp (Osram64610) was modulated with a mechanical chopper before passing the monochromator (Oriel, Cornerstone 130) for wavelength selection using a $2 \mathrm{~mm}$ diameter aperture to illuminate the active area. A calibrated Si cell was used as reference. Polymer solar cells were kept behind a quartz window in a nitrogen filled container.

\section{Data availability}

All relevant data in this study are available from the corresponding author upon request.

Received: 9 April 2019 Accepted: 15 May 2019

Published online: 28 June 2019

\section{References}

1. Threlfall, T. L. Analysis of organic polymorphs. A review. Analyst 120, 2435-2460 (1995)

2. Chung, H. \& Diao, Y. Polymorphism as an emerging design strategy for high performance organic electronics. J. Mater. Chem. C. 4, 3915-3933 (2016). 
3. He, T. et al. Single-crystal field-effect transistors of new Cl2-NDI polymorph processed by sublimation in air. Nat. Commun. 6, 5954 (2015).

4. Mattheus, C. C., de Wijs, G. A., de Groot, R. A. \& Palstra, T. T. M. Modeling the polymorphism of pentacene. J. Am. Chem. Soc. 125, 6323-6330 (2003).

5. Siegrist, T. et al. A polymorph lost and found: the high-temperature crystal structure of pentacene. Adv. Mater. 19, 2079-2082 (2007).

6. He, P. et al. Tuning the crystal polymorphs of alkyl thienoacene via solution self-assembly toward air-stable and high-performance organic field-effect transistors. Adv. Mater. 27, 825-830 (2015).

7. Steyrleuthner, R. et al. The role of regioregularity, crystallinity, and chain orientation on electron transport in a high-mobility n-type copolymer. J. Am. Chem. Soc. 136, 4245-4256 (2014).

8. Anthony, J. E. Functionalized acenes and heteroacenes for organic electronics. Chem. Rev. 106, 5028-5048 (2006).

9. Brinkmann, M. et al. Segregated versus mixed interchain stacking in highly oriented films of naphthalene diimide bithiophene copolymers. ACS Nano 6 , 10319-10326 (2012).

10. Tremel, K. et al. Charge transport anisotropy in highly oriented thin films of the acceptor polymer P(NDI2OD-T2). Adv. Energy Mater. 4, 1301659 (2014).

11. Fischer, F. S. U. et al. Highly crystalline films of PCPDTBT with branched side chains by solvent vapor crystallization: influence on opto-electronic properties. Adv. Mater. 27, 1223-1228 (2014).

12. Huang, C.-F. et al. Polymorphisms and morphological studies of a difluorobenzothiadiazole conjugated copolymer with $7.8 \%$ polymer solar cell efficiency. J. Mater. Chem. A 3, 3968-3974 (2015).

13. Lu, G., Li, L. \& Yang, X. Morphology and crystalline transition of poly(3butylthiophene) associated with its polymorphic modifications. Macromolecules 41, 2062-2070 (2008).

14. Poelking, C. \& Andrienko, D. Effect of polymorphism, regioregularity and paracrystallinity on charge transport in poly(3-hexylthiophene) [P3HT] nanofibers. Macromolecules 46, 8941-8956 (2013).

15. Fischer, F. S. U., Kayunkid, N., Trefz, D., Ludwigs, S. \& Brinkmann, M. Structural models of poly(cyclopentadithiophene-alt-benzothiadiazole) with branched side chains: impact of a single fluorine atom on the crystal structure and polymorphism of a conjugated polymer. Macromolecules 48, 3974-3982 (2015).

16. Zhong, Y., Biniek, L., Leclerc, N., Ferry, S. \& Brinkmann, M. Segregated versus disordered stacking in two low bandgap alternated copolymers for photovoltaic applications: impact of polymorphism on optical properties. Macromolecules 51, 4238-4249 (2018).

17. Schulz, G. L. et al. The PCPDTBT family: correlations between chemical structure, polymorphism, and device performance. Macromolecules 50, 1402-1414 (2017).

18. Noriega, R. et al. A general relationship between disorder, aggregation and charge transport in conjugated polymers. Nat. Mater. 12, 1038-1044 (2013).

19. Steyrleuthner, R. et al. Aggregation in a high-mobility n-type low-bandgap copolymer with implications on semicrystalline morphology. J. Am. Chem. Soc. 134, 18303-18317 (2012).

20. Cadby, A. J. et al. Film morphology and photophysics of polyfluorene. Phys. Rev. B 62, 15604-15609 (2000).

21. Grell, M. et al. Chain geometry, solution aggregation and enhanced dichroism in the liquidcrystalline conjugated polymer poly(9,9-dioctylfluorene). Acta Polym. 49, 439-444 (1998)

22. Peet, J., Brocker, E., Xu, Y. \& Bazan, G. C. Controlled $\beta$-Phase formation in poly(9,9-di-n-octylfluorene) by processing with alkyl additives. Adv. Mater. 20, $1882-1885$ (2008).

23. Köhler, A., Hoffmann, S. T. \& Bässler, H. An order-disorder transition in the conjugated polymer MEH-PPV. J. Am. Chem. Soc. 134, 11594-11601 (2012).

24. Yuan, Y. et al. Polymorphism and structural transition around $54^{\circ} \mathrm{C}$ in regioregular poly(3-hexylthiophene) with high crystallinity as revealed by infrared spectroscopy. Macromolecules 44, 9341-9350 (2011).

25. Lu, G. H., Li, L. G. \& Yang, X. N. Achieving perpendicular alignment of rigid polythiophene backbones to the substrate by using solvent-vapor treatment. Adv. Mater. 19, 3594-3598 (2007).

26. Meille, S. V. et al. Influence of molecular weight and regioregularity on the polymorphic behavior of poly(3-decylthiophenes). Macromolecules 30, 7898-7905 (1997).

27. Prosa, T. J., Winokur, M. J. \& McCullough, R. D. Evidence of a novel side chain structure in regioregular poly(3-alkylthiophenes). Macromolecules 29, 3654-3656 (1996).

28. Guo, Y., Wang, L., Han, Y., Geng, Y. \& Su, Z. Influence of molecular weight on polymorphs and temperature-induced structure evolution of regioregular poly (3-dodecylthiophene). Polym. Chem. 5, 1938-1944 (2014).

29. Panzer, F., Sommer, M., Bässler, H., Thelakkat, M. \& Köhler, A. Spectroscopic signature of two distinct $\mathrm{H}$-aggregate species in poly(3-hexylthiophene). Macromolecules 48, 1543-1553 (2015).

30. Hellmann, C. et al. Controlling the interaction of light with polymer semiconductors. Adv. Mater. 25, 4906-4911 (2013).
31. Heintges, G. H. L., Leenaers, P. J. \& Janssen, R. A. J. The effect of sidechain substitution and hot processing on diketopyrrolopyrrole-based polymers for organic solar cells. J. Mater. Chem. A 5, 13748-13756 (2017).

32. Ashraf, R. S. et al. Chalcogenophene comonomer comparison in small band gap diketopyrrolopyrrole-based conjugated polymers for high-performing field-effect transistors and organic solar cells. J. Am. Chem. Soc. 137 1314-1321 (2015).

33. Zoombelt, A. P., Mathijssen, S. G. J., Turbiez, M. G. R., Wienk, M. M. \& Janssen, R. A. J. Small band gap polymers based on diketopyrrolopyrrole. J. Mater. Chem. 20, 2240-2246 (2010).

34. Hendriks, K. H., Li, W., Wienk, M. M. \& Janssen, R. A. J. Small-bandgap semiconducting polymers with high near-infrared photoresponse. J. Am. Chem. Soc. 136, 12130-12136 (2014).

35. Spano, F. C. The spectral signatures of frenkel polarons in $\mathrm{H}-$ and $\mathrm{J}-$ Aggregates. Acc. Chem. Res. 43, 429-439 (2010).

36. Langeveld-Voss, B. M. W., Christiaans, M. P. T., Janssen, R. A. J. \& Meijer, E. W. Inversion of optical activity of chiral polythiophene aggregates by a change of solvent. Macromolecules 31, 6702-6704 (1998).

37. Panzer, F., Bässler, H. \& Köhler, A. Temperature induced order-disorder transition in solutions of conjugated polymers probed by optical spectroscopy. J. Phys. Chem. Lett. 8, 114-125 (2017).

38. Wang, Z. et al. Charge mobility enhancement for conjugated DPPselenophene polymer by simply replacing one bulky branching alkyl chain with linear one at each DPP unit. Chem. Mater. 30, 3090-3100 (2018).

39. Li, M. et al. Controlling the surface organization of conjugated donor-acceptor polymers by their aggregation in solution. Adv. Mater. 28 9430-9438 (2016)

40. Persson, N. E., Chu, P.-H., McBride, M., Grover, M. \& Reichmanis, E. Nucleation, growth, and alignment of poly(3-hexylthiophene) nanofibers for high-performance OFETs. Acc. Chem. Res. 50, 932-942 (2017).

41. Chen, Z. et al. Low band-gap conjugated polymers with strong interchain aggregation and very high hole mobility towards highly efficient thick-film polymer solar cells. Adv. Mater. 26, 2586-2591 (2014).

42. $\mathrm{Li}, \mathrm{M}$. et al. Impact of interfacial microstructure on charge carrier transport in solution-processed conjugated polymer field-effect transistors. Adv. Mater. 28, 2245-2252 (2016)

43. Back, J. Y. et al. Investigation of structure-property relationships in diketopyrrolopyrrole-based polymer semiconductors via side-chain engineering. Chem. Mater. 27, 1732-1739 (2015).

44. Tsao, H. N. et al. Ultrahigh mobility in polymer field-effect transistors by design. J. Am. Chem. Soc. 133, 2605-2612 (2011).

45. van Franeker, J. J. et al. Polymer solar cells: solubility controls fiber network formation. J. Am. Chem. Soc. 137,, 11783-11794 (2015).

46. Tauler, R., Smilde, A. \& Kowalski, B. Selectivity, local rank, three-way data analysis and ambiguity in multivariate curve resolution. J. Chemom. 9, 31-58 (1995).

47. de Juan, A., Jaumot, J. \& Tauler, R. Multivariate Curve Resolution (MCR). Solving the mixture analysis problem. Anal. Methods 6, 4964-4976 (2014).

48. Hess, B., Kutzner, C., van der Spoel, D. \& Lindahl, E. GROMACS 4: algorithms for highly efficient, load-balanced, and scalable molecular simulation. J. Chem. Theor. Comput. 4, 435-447 (2008).

49. Berendsen, H. J. C., Postma, J. P. M., van Gunsteren, W. F., DiNola, A. \& Haak, J. R. Molecular dynamics with coupling to an external bath. J. Chem. Phys. 81, 3684-3690 (1984).

50. Bussi, G., Donadio, D. \& Parrinello, M. Canonical sampling through velocity rescaling. J. Chem. Phys. 126, 014101 (2007).

51. Oosterhout, S. D. et al. Mixing behavior in small molecule:fullerene organic photovoltaics. Chem. Mater. 29, 3062-3069 (2017).

\section{Acknowledgements}

This project has received funding from the European Union's Horizon 2020 research and innovation program under the Marie Skłodowska-Curie grant agreement No. 747422 , from the Netherlands Organisation for Scientific Research $(016$. Veni.192.106), from the Chinese Academy of Sciences (Grant No. XDB12020200), and from King Abdullah University of Science and Technology (KAUST). The research also received funding from the European Community's Seventh Framework Programme (FP7/2007-2013) (Grant Agreement no. 607585, project OSNIRO), the European Research Council (ERC Grant Agreement No. 33903), and the Ministry of Education, Culture, and Science (Gravity program 024.001.035). T.M. acknowledges the Foundation for Polish Science co-financed by the European Union under the European Regional Development Fund (First TEAM/2017-3/26). The authors acknowledge Beamline 9 of the DELTA electron storage ring in Dortmund for GIWAXS measurements. The authors thank Mateusz Brzezinski for the discussion about GIWAXS analysis, and Christ Weijtens for UPS measurements. 


\section{Author contributions}

The idea of this work is from M.L. and R.A. J.J.; P.J.L. and G.H. L.H. synthesized the polymer; P.J.L. measured GPC; M.L. performed AFM; M.L. conducted steady-state spectroscopic measurements, and M.M.W., S.C.J.M. and R.A. J.J. engaged in subsequent analysis; A.H.B. conducted time-resolved photoluminescence experiments and F.L. engaged in the subsequent analysis; L.N. and Y.Y. carried out the MD simulation; T.M. measured GIWAXS, and M.L., and W.P. engaged in subsequent analysis; M.L. and G.H. L.H. measured DSC; M.L. fabricated OFETs and OPVs, as well as corresponding device characterization. R.A. J.J. supervised the project. All authors commented on the data and co-wrote the manuscript.

\section{Additional information}

Supplementary Information accompanies this paper at https://doi.org/10.1038/s41467019-10519-z.

Competing interests: The authors declare no competing interests.

Reprints and permission information is available online at http://npg.nature.com/ reprintsandpermissions/
Peer review information: Nature Communications thanks the anonymous reviewers for their contribution to the peer review of this work.

Publisher's note: Springer Nature remains neutral with regard to jurisdictional claims in published maps and institutional affiliations.

(c) (i) Open Access This article is licensed under a Creative Commons Attribution 4.0 International License, which permits use, sharing, adaptation, distribution and reproduction in any medium or format, as long as you give appropriate credit to the original author(s) and the source, provide a link to the Creative Commons license, and indicate if changes were made. The images or other third party material in this article are included in the article's Creative Commons license, unless indicated otherwise in a credit line to the material. If material is not included in the article's Creative Commons license and your intended use is not permitted by statutory regulation or exceeds the permitted use, you will need to obtain permission directly from the copyright holder. To view a copy of this license, visit http://creativecommons.org/ licenses/by/4.0/.

(C) The Author(s) 2019 